\title{
Construct Validity of a Korean Version of the WHOQOL-BREF and EQ-5D Questionnaire Applied on Various Cancer Survivors Undergoing Palliative Rehabilitation Programs
}

\author{
Bong-sam Choi, PT, MPH, PhD \\ Department of Physical Therapy, College of Health and Welfare, Woosong University, Daejeon, Korea
}

\author{
Article Info \\ Received June 29, 2020 \\ Revised July 16, 2020 \\ Accepted July 29, 2020 \\ Corresponding Author \\ Bong-sam Choi \\ E-mail: bchoi@wsu.ac.kr \\ https://orcid.org/0000-0002-0165-4941
}

\section{Key Words}

Cancer survivors

Health related quality of life

Palliative medicine

Psychometrics
Background: Although the original version of the health-related quality of life (HRQOL) questionnaires are found to be acceptable, the cross-culturally adapted versions may not be comparable to their original version.

Objects: To examine dimensionality and construct validity of two Korean versions of the brief version of the World Health Organization Quality of Life (WHOQOL-BREF) and EuroQOL-5 dimension (EQ-5D) questionnaires.

Methods: A total of 77 cancer survivors undergoing palliative rehabilitation programs from two rehabilitation institutes was recruited from April 16, 2018 to June 26, 2019. The WHOQOL-BREF and the EQ-5D were filled out by the various cancer survivors following a particular session of rehabilitation programs. The scores were analyzed with Winsteps Rasch analysis computer program using the rating scale model. Rasch fit statistics were used to determine the dimensionality and the item difficulty calibrations of WHOQOL-BREF and EQ-5D.

Results: All items except two, negative feeling, need treatment function and pain prevent activity (mean square $[\mathrm{MnSq}]=2.42,1.82$ and 2.51, respectively), were found to be acceptable, while two items of the EQ-5D, anxiety/depression and self-care, were misfit (infit MnSq = 1.65 and 0.38 , respectively). Item difficulty calibrations of WHOQOL-BREF match person ability measures (i.e., HRQOL) fairly well. However, the person ability distribution showed obvious ceiling effects for EQ-5D. All items of EQ-5D were appeared to be less challenged in comparison with those of WHOQOL-BREF.

Conclusion: Item-level analysis using the Rasch model supports the quality of culturally adapted items used to measure the HRQOL one exception; that is, whether or not to include misfit items as part of the HRQOL measurements. Additionally, cancer survivors undergoing palliative rehabilitation programs appear to have more of a tendency to view the EQ-5D items as being more challenging than the WHOQOL-BREF.

\section{INTRODUCTION}

The latest worldwide cancer statistics estimated 9.6 million deaths in 2018 and reported an increasing burden of care resulting from various cancers. It is one of the most costly conditions of global population with the economic impact exceeding 1.16 trillion dollars in 2010 in the United States [1]. According to the national cancer control institute in Korea, the average 5-year survival rate of individuals with various cancer conditions increased about 69.4\% in 2017 [2]. Despite potential improvements in the survivorship, these cancer survivors are generally susceptible to have some limitations in their healthrelated quality of life (HRQOL). Yet they are three times more likely to report their limitations during the course of survivorship [3].

In general, palliative rehabilitation program (PRP) controlling negative symptoms includes approaches primarily intended to enhance the HRQOL of the cancer survivors. This has been well evidenced in many peer-reviewed journals [37]. The primary goals of PRP are now acknowledged to include concept of the HRQOL addressing the palliative care of cancer survivors [8,9]. The HRQOL questionnaires often include global 
ratings of health status and multidimensional status of HRQOL. These measures often deal with a broad spectrum of health concepts and are intended to provide scores that are sensitive to all ranges of the concept being measured. By contrast, some measures are designed to assess the aspects of health status resulting from a particular pathology and to view the attribution of functional limitations to the specific condition [4,5].

Most commonly used HRQOL questionnaires, if not all, have often been noted for being psychometrically narrow, whereas the HRQOL is a multidimensional health-oriented concept encompassing aspects of health or health care, such as functioning and symptoms [8]. Therefore, the outcome measures require underlying constructs reflecting particular goals of the types of rehabilitation programs, such as improving the HRQOL before death, controlling symptoms and supporting other factors [10]. During the course of cancer survivorship, it is imperative to monitor how the programs impact the HRQOL and how optimally assess any variation of the HRQOL over time in suitable manner.

Of the most widely accepted HRQOL questionnaires, the brief version of the World Health Organization Quality of Life (WHOQOL-BREF) and EuroQOL-5 dimension descriptive system (EQ-5D) are considered to be optimal for cancer survivors in many peer reviewed journals in regards to construct validity and psychometric adequacy [7,11-13]. Cross-culturally adapted versions of them were validated in many peer-reviewed journals and proved to be reliable, also responsive [12,14,15]. Since these two questionnaires were simultaneously developed in many countries, such approaches offered intriguing a possibilities warranting the cross-cultural adaptation related validity of the measure. However, as demand for the measure grew in countries where no language version of the measure existed, it naturally developed many translated versions. Accordingly, in such cases, the most appropriate version has been used as a starting point for the adaptation and updated by many authors.

Numerous classical test theory (CTT)-based HRQOL measures for cancer survivors have been developed and crossculturally adapted into other languages. With almost endless array of translated HRQOL measures attempted to maintain psychometrical properties after cross-cultural adaptation procedure, often there is little distinction between the versions of the measures [13-16]. While designed to assess on-going difficulties on the dimension of HRQOL, cross-culturally adapted versions of the measures often fail to maintain the dimensions referred to in the original version [6]. An evidence was obtained for a multidimensional perspective of the HRQOL in regards to construct validity. That is, the measurement structure accounts for construct validity in item responses either due to factors underlying the data or mistakenly treated latent traits they are intended to measure [6].

In addition, despite the myriad existing HRQOL measures, most investigators are often faced with a prevailing challenge of selecting an optimal measure. Although most CTT-based questionnaires have adequate psychometric properties, they may be insensitive either to a broad range of HRQOL level or to the actual variations that result from particular cancer experiences. One of the reasons is that these questionnaires are commonly developed to target the average person, hence the questionnaires are more likely to be sensitive at center than at both extremes in the ability range [17]. Consequently, these measures often demonstrate ceiling effects when these measures are administered to persons with low ability score and floor effects when administered to persons with high ability score [18-20].

The purpose of this study was to compare dimensionality and construct validity of two Korean version of WHOQOLBREF and EQ-5D questionnaires.

\section{MATERIALS AND METHODS}

\section{Subjects}

A total of 77 cancer survivors undergoing palliative rehabilitation programs from an oriental medicine hospital in Busan and a rehabilitation hospital in Daejeon, Republic of Korea were recruited from April 16, 2018 to June 26, 2019. Potential subjects consisted of all appropriate clients undergoing the palliative rehabilitation program at the participating sites during the period. All participants received detailed information on the present study including informed consent and potential conflict of interests of which non-participation in the study may not influence whether the palliative rehabilitation programs terminated. Subjects were asked to sign an informed consent and the Korean version of the WHOQOL-BREF and the EQ-5D approved by the Institutional Review Board of College of Health and Welfare, Woosong University (approval No. 1041549-190114-SB-70). The Korean versions of the WHOQOL-BREF and the EQ-5D [21,22] were administered 
(i.e, WHOQOL-BREF was followed by EQ-5D) upon the last entry of the first bout of rehabilitation program into physical therapy service and was subsequently requested from the survivors at discharge following the completion of the program. Of the participants who completed two measures, more than 35 percent were male $(n=35)$ and less than 65 percent $(n=$ 42) were female with an average age of 52.9 ranged from 35 through 77. In addition, forty-six percent of the participants were diagnosed with breast cancer and fifty-four percent were diagnosed with various cancers (i.e., cancers on colon, lung, stomach, pancreas, kidney, prostate, liver, ovary, lymphatic system related, tonsil, bile duct and brain).

\section{Data Analysis}

Scores were analyzed with the Winsteps software program version 3.57.2 (Wisteps.com, Chicago, IL, USA) using a rating scale model $[23,24]$. Rasch fit statistics and item difficulty calibrations were examined to determine the dimensionality of the Korean version of the WHOQOL-BREF. The construct validity of the Korean version of the WHOQOL-BREF was visually examined using the person-item map. The Rasch model transforms raw scores into the estimate of person ability (i.e., the level of HRQOL) and item difficulty (i.e., more or less challenging items) in logits. All descriptive statistics were calculated using the SPSS software version 25.0 (IBM Co., Armonk, NY, USA).

The Winsteps software program provides goodness-of-fit statistics for each item and subject. These fit statistics were examined to detect items that did not fit the Rasch rating scale model criterion of dimensionality (or unidimensionality). The Infit and outfit mean square $(\mathrm{MnSq}) \geq 1.4$ and $\leq 0.6$ and a standardized score greater than 2.0 were considered to be misfit, which is an indication that the particular item or survivor responded in unexpected ways [23]. The unexpected response indicated by the fit statistics suggests that the particular item is measuring another construct rather than the HRQOL. The program also generates a log odds unit (i.e., logit) scale to present the survivor's level of HRQOL and item difficulty. The logit scale is based on the probability of getting success over the probability of getting failure on a response category of each item. The logical fashion of the analysis places test items along a continuum of the most to the least in difficulty. The hierar-

Table 1. Fit statistics of the WHOQOL-BREF in descending order of difficulty

\begin{tabular}{|c|c|c|c|c|c|}
\hline Items & Difficulty (logits) & Infit MnSq & ZSTD & Outfit MnSq & ZSTD \\
\hline WHOQOL 26 negative feeling & 0.76 & 2.42 & 5.3 & 2.55 & 5.7 \\
\hline WHOQOL 14 leisure activity & 0.70 & 0.92 & -0.4 & 0.92 & -0.4 \\
\hline WHOQOL 21 sex life & 0.45 & 0.89 & -0.4 & 0.98 & 0 \\
\hline WHOQOL 9 healthy environment & 0.44 & 0.95 & -0.2 & 1.00 & 0.1 \\
\hline WHOQOL 4 need treatment to function & 0.37 & 1.82 & 3.9 & 2.20 & 5.2 \\
\hline WHOQOL 3 pain prevent activity & 0.36 & 2.51 & 5.5 & 3.21 & 6.9 \\
\hline WHOQOL 18 work capacity & 0.34 & 0.64 & -2.2 & 0.63 & -2.1 \\
\hline WHOQOL 2 general health & 0.32 & 1.06 & 0.4 & 1.06 & 0.6 \\
\hline WHOQOL 23 living place & 0.15 & 0.86 & -0.7 & 0.86 & -0.7 \\
\hline WHOQOL 11 bodily appearance & 0.13 & 0.73 & -1.4 & 0.73 & -1.4 \\
\hline WHOQOL 12 enough money & 0.12 & 1.05 & 0.3 & 1.16 & 0.7 \\
\hline WHOQOL 5 enjoy life & 0.03 & 0.73 & -1.5 & 0.75 & -1.4 \\
\hline WHOQOL 10 energy for daily life & -0.01 & 0.69 & -1.6 & 0.61 & -1.7 \\
\hline WHOQOL 16 sleep & -0.04 & 1.29 & 1.6 & 1.31 & 1.7 \\
\hline WHOQOL 15 get around & -0.10 & 0.70 & -1.9 & 0.67 & -2.0 \\
\hline WHOQOL 13 available information & -0.14 & 0.76 & -1.4 & 0.75 & -1.4 \\
\hline WHOQOL 22 support from friend & -0.16 & 0.98 & 0 & 0.98 & 0 \\
\hline WHOQOL 17 satisfaction on daily life & -0.22 & 0.69 & -1.8 & 0.69 & -1.8 \\
\hline WHOQOL 19 self-satisfaction & -0.22 & 0.75 & -1.3 & 0.75 & -1.4 \\
\hline WHOQOL 20 personal relationship & -0.23 & 0.78 & -1.3 & 0.77 & -1.3 \\
\hline WHOQOL 1 overall quality of life & -0.26 & 0.85 & -0.7 & 0.87 & -0.7 \\
\hline WHOQOL 24 health service & -0.28 & 0.84 & -0.8 & 0.81 & -1.0 \\
\hline WHOQOL 8 feel safe for daily life & -0.47 & 0.71 & -1.6 & 0.72 & -1.5 \\
\hline WHOQOL 25 transport & -0.54 & 0.80 & -1.0 & 0.79 & -1.1 \\
\hline WHOQOL 6 meaningful life & -0.72 & 0.83 & -0.8 & 0.83 & -0.8 \\
\hline WHOQOL 7 ability to concentrate & -0.78 & 0.83 & -0.9 & 0.83 & -0.8 \\
\hline
\end{tabular}

WHOQOL-BREF, brief version of the World Health Organization Quality of Life; MnSq, mean square standardized residuals; ZSTD, Z score standardized. 
chical order of item difficulty provides a means to examine the construct validity of the instrument [23].

To determine how well the EQ-5D and the WHOQOL-BREF represent the construct of HRQOL, data were analyzed by exploratory factor analysis. A correlation matrix in which intercorrelations between individual test items was used respectively. The dimensionality of the matrix can be reduced by inspecting the items highly correlating with a group of other items. However, the matrix may represent low correlations with items outside of that group [25]. In a nutshell, these items with high correlations could measure the trait being measured well, which is called a "factor". The obtained factor creates a new dimension visualizing classification axes along with test items can be plotted. This projection of the scores of the original test items on the factor leads to two concepts: factor score and loadings. Factor scores are the scores of a subject on a particular factor, while the loadings are the correlations of the original test items with a factor. The factor loadings are now especially useful in determining the substantive importance of a particular item to a factor [25]. A rotated factor analysis was used to compute maximizing the loadings from the original factor matrix with eigenvalues greater than 1 . In addition, greater than 0.40 as a significant loading was used.

Table 2. Fit statistics of the EQ-5D in descending order of difficulty

\begin{tabular}{lcccrr}
\hline \multicolumn{1}{c}{ Items } & Difficulty (logits) & Infit MnSq & ZSTD & Outfit MnSq & ZSTD \\
\hline EQ-5D 4 - pain/discomfort & 0.72 & 0.95 & -0.1 & 1.22 & 0.9 \\
EQ-5D 3 - usual activities & 0.61 & 0.62 & -1.9 & -2.52 & 1.04 \\
EQ-5D 1 - mobility & 0.06 & 0.82 & -0.6 & 1.69 & 0.2 \\
EQ-5D 5 - anxiety/depression & 0.02 & 1.65 & -2.7 & 0.22 & -1.4 \\
EQ-5D 2 - self-care & -1.41 & 0.38 & & \\
\hline
\end{tabular}

EQ-5D, EuroQOL-5 dimension; MnSq, mean square standardized residuals; ZSTD, Z score standardized.

Table 3. Factor loadings for the WHOQOL-BREF

\begin{tabular}{|c|c|c|c|c|c|c|c|}
\hline \multirow{2}{*}{ Items } & \multicolumn{7}{|c|}{ Factor loadings } \\
\hline & 1 & 2 & 3 & 4 & 5 & 6 & 7 \\
\hline WHOQOL1 - overall quality of life & 0.604 & -0.204 & -0.048 & -0.120 & 0.346 & 0.399 & 0.098 \\
\hline WHOQOL2 - general health & 0.540 & -0.513 & -0.250 & -0.013 & 0.262 & -0.166 & -0.035 \\
\hline WHOQOL3 - pain prevent activity & -0.495 & 0.222 & 0.220 & 0.343 & 0.296 & 0.113 & 0.326 \\
\hline WHOQOL4 - need treatment to function & -0.184 & 0.247 & 0.785 & 0.094 & -0.066 & 0.163 & 0.043 \\
\hline WHOQOL5 - enjoy life & 0.716 & 0.016 & 0.360 & -0.034 & 0.287 & 0.104 & 0.031 \\
\hline WHOQOL6 - meaningful life & 0.573 & -0.052 & 0.599 & -0.306 & 0.008 & -0.062 & 0.158 \\
\hline WHOQOL7 - ability to concentrate & 0.598 & 0.306 & 0.155 & 0.483 & -0.055 & -0.162 & 0.011 \\
\hline WHOQOL8 - feel safe for daily life & 0.707 & -0.145 & 0.041 & 0.177 & 0.209 & 0.201 & -0.054 \\
\hline WHOQOL9 - healthy environment & 0.483 & 0.511 & -0.275 & 0.383 & -0.150 & 0.285 & -0.004 \\
\hline WHOQOL10 - energy for daily life & 0.754 & 0.014 & 0.016 & 0.239 & 0.310 & -0.222 & 0.000 \\
\hline WHOQOL11 - bodily appearance & 0.705 & 0.386 & 0.053 & 0.065 & 0.091 & -0.308 & -0.082 \\
\hline WHOQOL12 - enough money & 0.536 & 0.528 & -0.118 & 0.038 & -0.154 & 0.274 & -0.110 \\
\hline WHOQOL13 - available information & 0.702 & 0.246 & 0.074 & -0.190 & -0.301 & 0.206 & -0.222 \\
\hline WHOQOL14 - leisure activity & 0.605 & -0.156 & 0.059 & 0.005 & -0.047 & 0.383 & -0.261 \\
\hline WHOQOL15 - get around & 0.778 & -0.232 & -0.151 & 0.134 & 0.097 & 0.006 & -0.133 \\
\hline WHOQOL16 - sleep & 0.242 & -0.466 & -0.078 & 0.199 & -0.398 & 0.220 & 0.546 \\
\hline WHOQOL17 - satisfaction on daily life & 0.760 & -0.355 & -0.018 & 0.358 & 0.145 & -0.071 & 0.055 \\
\hline WHOQOL18 - work capacity & 0.826 & -0.088 & 0.029 & 0.248 & 0.055 & 0.136 & -0.045 \\
\hline WHOQOL19 - self satisfaction & 0.703 & -0.257 & 0.366 & -0.079 & -0.235 & -0.265 & -0.013 \\
\hline WHOQOL20 - personal relationship & 0.628 & 0.180 & 0.144 & 0.068 & -0.184 & -0.404 & -0.120 \\
\hline WHOQOL21 - sex life & 0.560 & -0.169 & -0.179 & -0.311 & -0.203 & 0.027 & -0.372 \\
\hline WHOQOL22 - support from friend & 0.435 & -0.012 & 0.237 & -0.657 & 0.100 & 0.192 & 0.124 \\
\hline WHOQOL23 - living place & 0.587 & 0.226 & -0.386 & -0.310 & 0.002 & -0.080 & 0.309 \\
\hline WHOQOL24 - health service & 0.574 & 0.337 & -0.192 & -0.359 & 0.158 & -0.260 & 0.269 \\
\hline WHOQOL25 - transport & 0.639 & 0.397 & -0.240 & -0.139 & -0.012 & 0.069 & 0.330 \\
\hline WHOQOL26 - negative feeling & -0.567 & 0.306 & -0.034 & -0.126 & 0.549 & 0.085 & -0.242 \\
\hline
\end{tabular}

WHOQOL-BREF, brief version of the World Health Organization Quality of Life. 


\section{RESULTS}

Rasch fit statistics were examined to determine the dimensionality of the Korean version of WHOQOL-BREF and EQ-5D. Using the criterion of misfit as MnSq > 1.4 and ZSTD > 2.0, the Table 1 presents item measures, infit/outfit statistics for the 26 WHOQOL-BREF items. All items, except the negative feeling, need treatment function and pain prevent activity items, show acceptable fit statistics (Table 1). For the EQ-5D items, all items, except the anxiety/depression and self-care items being slightly misfit, present acceptable fit statistics (Table 2).

To further examine the dimensionality of the WHOQOLBREF, exploratory factor analysis was applied to the WHOQOL-BREF questionnaires. Table 3 presents factor loadings of the WHOQOL-BREF with a total of 7 constructs addressing HRQOL domains. Furthermore, the factor analysis revealed that a total of 22 items loaded on factor 1 and other items loaded either one or two factors (Table 3). Unlike the underlying 4 constructs originally designed, the factor analysis did not support the 4 constructs for the WHOQOL-BREF.

The person-item match analysis presents the relationship between the survivors' various levels of HRQOL and item difficulty measures in logits for the two questionnaires. For the WHOQOL-BREF questionnaire, item difficulty calibrations were fairly well matched to the survivors' levels of HRQOL throughout the whole range with some ceiling and floor effects. However, the EQ-5D showed a serious gap which was unable to effectively measure most cancer survivors at the medium and high levels of HRQOL. The items of the EQ-5D were only able to measure the individuals at the lower levels of HRQOL (Figure 1).

\section{DISCUSSION}

Construct validity of two well-established HRQOL questionnaires was tested using the Rasch-fit statistics and exploratory factor analysis. Overall, the Korean version of the WHOQOLBREF showed acceptable construct validity except for few erratic items (i.e., negative feeling, need treatment function and pain prevent activity items of the WHOQOL-BREF, Anxiety/depression and self-care items of the EQ-5D with slight high/low fit). The exploratory factor analysis revealed that a total of 24 items loaded on factor 1 and other items loaded either one or two factors. Consequently, the WHOQOL-BREF showed nearly

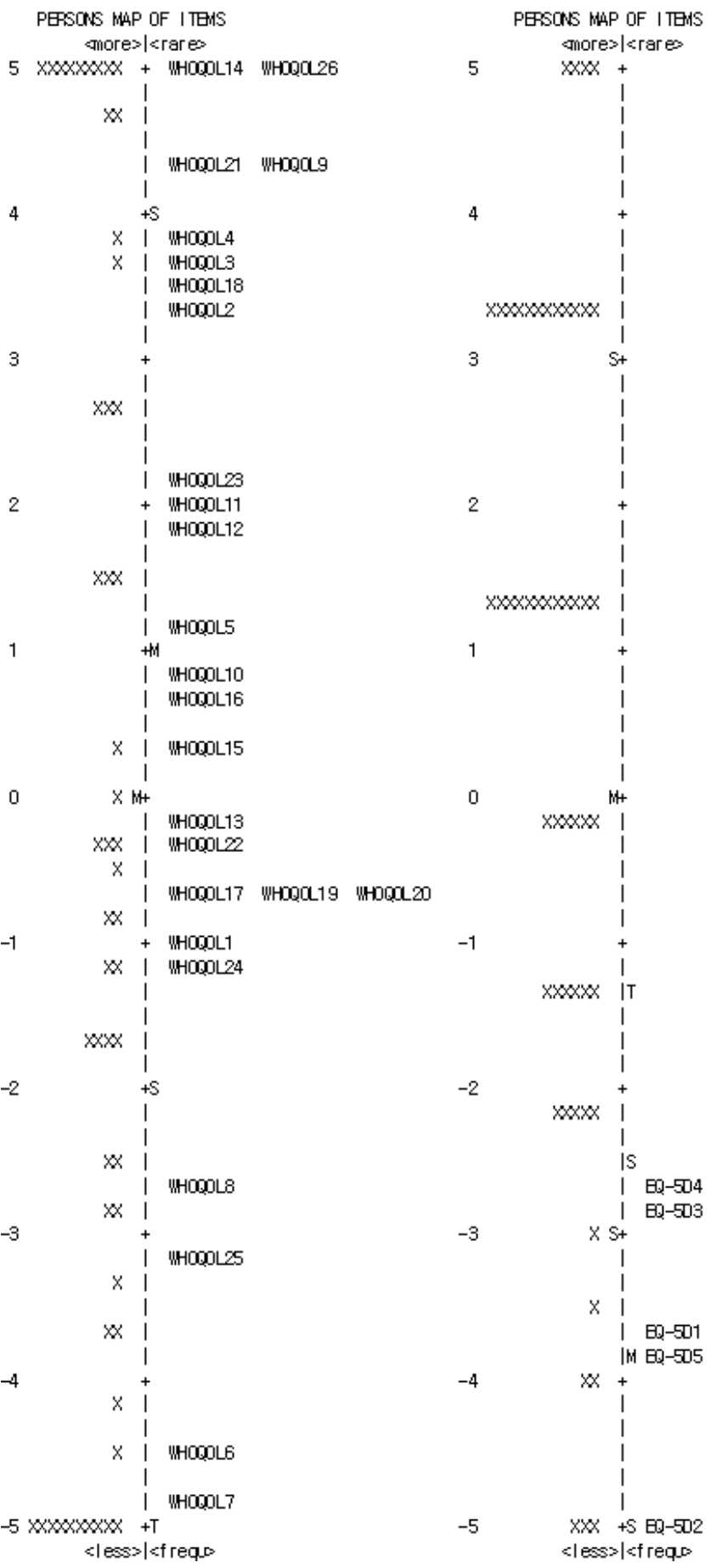

Figure 1. Person-item match of the Korean version of the WHOQOLBREF. The graph represents the relationship among the survivors' level of HRQOL and item difficulty calibrations in logits. Each "\#" on the left side of each map represents two subjects, with the \#s at the bottom of the scale representing individuals of low HRQOL level and \#s at the top of the scale representing individuals with high HRQOL level. WHOQOLBREF, brief version of the World Health Organization Quality of Life; $H R Q O L$, health-related quality of life; EQ-5D, EuroQOL-5 dimension.

a single underlying construct. Furthermore, item difficulty hierarchy of the WHOQOL-BREF was fairly well matched to the HRQOL levels of the survivors throughout the whole range 
despite some ceiling and floor effects. However, item difficulty hierarchy of the EQ-5D showed a serious gap which was unable to measure all cancer survivors at the medium and high QOL levels. The items of the EQ-5D were only able to measure the individuals at the lower levels of HRQOL.

Items fit the Rasch model based on the probability of getting a rating on each item. For example, a cancer survivor with high level of HRQOL would be expected to have more difficulty on challenging items (i.e., WHOQOL-BREF item 26 through item 2 in Table 1) than less challenging items (i.e., WHOQOLBREF item 7 through item 22 in Table 1). Similarly, a cancer survivor with low level of HRQOL would be expected to have even more difficulty on those challenging items than the survivors with high level of HRQOL. For the EQ-5D questionnaire with 5 items, the pain/discomfort item is the most challenging, while self-care item is the least challenging. Likewise, a survivor who is having difficulty on the self-care item would be expected to have more difficulty on the pain/discomfort item. However, these logical fashions are not demonstrated by the self-care item of the EQ-5D questionnaire as well as the negative feeling and pain prevent activity items of the WHOQOLBREF (i.e., misfit items).

The World Health Organization (WHO) defines QOL as "individuals' perception of their position in life in the context of the culture and value systems in which they live and in relation to their goals, expectations, standards and concerns" [26]. Although the multidimensional concept of QOL has increasingly become a focus in the fields of health care, it is not clearly defined. The WHOQOL-BREF, a brief version of QOL questionnaire developed by the $\mathrm{WHO}$, provides insights into the impact of all aspects on QOL as well as on Health-Related QOL. In an effort to focus on the assessment of health and QOL, the term, health-related QOL is now widely accepted and used. The HRQOL, in general, can be impacted by the notion of which presence of particular disease does not inevitably mean poor QOL [6]. The implication of this is that various cancer survivors with such an experience of cancer related conditions may perceive their HRQOL as better than healthy individuals as long as palliative rehabilitation program goes successfully. Thus, it is advisable for clinicians to continually monitor their status of the HRQOL.

The construct of HRQOL questionnaire commonly changes and is unable to maintain the dimensions referred to in the original measures when a questionnaire is cross-culturally adapted [6]. Traditionally, investigators establish construct validity of a measure by correlating it with a number of other measures by inspecting the pattern of the correlations, in which the measure is associated with these variables in predictable ways [27]. However, a previous study, in a review of dimensionality problems with versions of the WHOQOL-BREF questionnaire, encouraged using item level analysis in evaluating dimensionality issues for validation procedure for the instrument $[6,24,28]$. The authors indicated that the nature of sample dependence often causes the dimensionality change which was explained as a dynamic construct model. That is, when translating a questionnaire into other languages, the questionnaire is more likely to be subject to change over time. To overcome this psychometric property limitation, item level analysis using item response theory is recommended to be used by focusing on the items of a questionnaire instead of the questionnaire as a whole [24]. This study focused on items of the two well-known HRQOL questionnaires and attempted to determine whether the latent structure accounted for a single construct purported to measure by the items can be confirmed.

The present study carries some inherent limitations because the Rasch analysis was applied to a possible multidimensional structure of the HRQOL and exploratory factor analysis was applied without optimal sample sizes. It is unknown whether the determination in the fit statistics and the factor loadings was optimal. In addition, the present study includes the misfit items for all analyses due to the items' critical roles within the questionnaires. In addition, the study subjects with various stages of cancer related conditions were recruited from two institutions where different palliative rehabilitation programs were carried. These factors could make substantial differences towards the levels of HRQOL. Future research is needed to investigate the effects of excluding the problematic items to confirm a single construct of the HRQOL.

\section{CONCLUSIONS}

Item level analyses using the Rasch model (1-parameter item response theory model) supports some psychometric properties of culturally adapted versions of the WHOQOL-BREF and the EQ-5D except two erratic items for each questionnaire. Whether or not to include the misfit items as part of the two questionnaires, cancer survivors undergoing palliative rehabilitation programs appear to have more of a tendency to view the 
WHOQOL-BREF items as being more challenging than the EQ$5 \mathrm{D}$.

\section{ACKNOWLEDGEMENTS}

This research is based on the support of 2020 Woosong University Academic Research Funding.

\section{CONFLICTS OF INTEREST}

No potential conflict of interest relevant to this article was reported.

\section{REFERENCES}

1. World Health Organization. Cancer. World Health Organization [Internet]. Geneva: 2018 Sep 12 [cited 2020 Jul 12]. Available from: https://www.who.int/news-room/fact-sheets/ detail/cancer.

2. Kang DW, Lee EY, An KY, Min J, Jeon JY, Courneya KS. Associations between physical activity and comorbidities in Korean cancer survivors. J Cancer Surviv 2018;12(4):441-9.

3. Oliveira IS, da Cunha Menezes Costa L, Fagundes FR, Cabral CM. Evaluation of cross-cultural adaptation and measurement properties of breast cancer-specific quality-of-life questionnaires: a systematic review. Qual Life Res 2015;24(5):117995.

4. Caplan N, Robson H, Robson A, Kelly M, Wilkes G. Changes in health-related quality of life (EQ-5D) dimensions associated with community-based musculoskeletal physiotherapy: a multi-centre analysis. Qual Life Res 2018;27(9):2373-82.

5. Efthymiadou 0, Mossman J, Kanavos P. Differentiation of health-related quality of life outcomes between five disease areas: results from an international survey of patients. Int J Technol Assess Health Care 2018;34(5):498-506.

6. Perera HN, Izadikhah Z, O'Connor P, Mcllveen P. Resolving dimensionality problems with WHOQOL-BREF item responses. Assessment 2018;25(8):1014-25.

7. Skevington SM, Lotfy M, O'Connell KA; WHOQOL Group. The World Health Organization's WHOQOL-BREF quality of life assessment: psychometric properties and results of the international field trial. A report from the WHOQOL group. Qual Life Res 2004;13(2):299-310.

8. Kaasa S, Loge JH. Quality of life in palliative care: principles and practice. Palliat Med 2003;17(1):11-20.

9. Desai MJ, Kim A, Fall PC, Wang D. Optimizing quality of life through palliative care. J Am Osteopath Assoc 2007;107(12 Suppl 7):ES9-14.

10. Avoine-Blondin J, Parent V, Lahaye M, Humbert N, Duval M, Sultan S. Identifying domains of quality of life in children with cancer undergoing palliative care: a qualitative study with professionals. Palliat Support Care 2017;15(5):565-74.

11. Khan I, Morris S, Pashayan N, Matata B, Bashir Z, Maguirre J. Comparing the mapping between EQ-5D-5L, EQ-5D-3L and the EORTC-QLQ-C30 in non-small cell lung cancer patients. Health Qual Life Outcomes 2016;14:60.

12. Payakachat N, Ali MM, Tilford JM. Can the EQ-5D detect meaningful change? A systematic review. Pharmacoeconomics 2015;33(11):1137-54.

13. Skevington SM, Sartorius N, Amir M. Developing methods for assessing quality of life in different cultural settings. The history of the WHOQOL instruments. Soc Psychiatry Psychiatr Epidemiol 2004;39(1):1-8.

14. Guillemin F, Bombardier C, Beaton D. Cross-cultural adaptation of health-related quality of life measures: literature review and proposed guidelines. J Clin Epidemiol 1993;46(12):141732.

15. Schmidt S, Bullinger M. Current issues in cross-cultural quality of life instrument development. Arch Phys Med Rehabil 2003;84(4 Suppl 2):S29-34.

16. Escobar A, Trujillo-Martín Mdel M, Rueda A, Pérez-Ruiz E, Avis NE, Bilbao A. Cross-cultural adaptation, reliability and validity of the Spanish version of the Quality of Life in Adult Cancer Survivors (QLACS) questionnaire: application in a sample of short-term survivors. Health Qual Life Outcomes 2015;13:182.

17. Jette AM, Haley SM, Ni P. Comparison of functional status tools used in post-acute care. Health Care Financ Rev 2003; 24(3):13-24.

18. McHorney CA. Health status assessment methods for adults: past accomplishments and future challenges. Annu Rev Public Health 1999;20:309-35.

19. Liang MH, Lew RA, Stucki G, Fortin PR, Daltroy L. Measuring clinically important changes with patient-oriented questionnaires. Med Care 2002;40(4 Suppl):II45-51.

20. Speyer R, Kim JH, Doma K, Chen YW, Denman D, Phyland D, et al. Measurement properties of self-report questionnaires on health-related quality of life and functional health status in 
dysphonia: a systematic review using the COSMIN taxonomy. Qual Life Res 2019;28(2):283-96.

21. Kim SH, Kim HJ, Lee SI, Jo MW. Comparing the psychometric properties of the EQ-5D-3L and EQ-5D-5L in cancer patients in Korea. Qual Life Res 2012;21(6):1065-73.

22. Min SK, Kim KI, Lee CI, Jung YC, Suh SY, Kim DK. Development of the Korean versions of WHO Quality of Life scale and WHOQOL-BREF. Qual Life Res 2002;11(6):593-600.

23. Bond TG, Fox CM. Applying the Rasch model: fundamental measurement in the human sciences. Mahwah: Lawrence Erlbaum; 2001;23-8.

24. Young TA, Rowen D, Norquist J, Brazier JE. Developing preference-based health measures: using Rasch analysis to generate health state values. Qual Life Res 2010;19(6):907-17.

25. Ledesma RD, Valero-Mora P, Macbeth G. The Scree test and the number of factors: a dynamic graphics approach. Span J Psychol 2015;18:E11.

26. World Health Organization. Programme on mental health: WHOQOL user manual. Geneva: World Health Organization; 1998;48-9.

27. Westen D, Rosenthal R. Quantifying construct validity: two simple measures. J Pers Soc Psychol 2003;84(3):608-18.

28. Danielsen AK, Pommergaard HC, Burcharth J, Angenete E, Rosenberg J. Translation of questionnaires measuring health related quality of life is not standardized: a literature based research study. PLoS One 2015;10(5):e0127050. 